would also be advisable if the dispensing of such prescription were not repeated without the written sanction of the medical man. The frequency of cases of veronal poisoning in this country has caused His Majesty's Privy Council to approve of the following addition to the Poisons and Pharmacy Act of 1908: that "diethyl barbituric acid, and other alkyl, aryl, or metallic derivatives of barbituric acid, whether described as veronal, proponal, medinal, or by any other trade name, mark, or designation, and all poisonous urethanes and ureides," ought to be deemed poisons within the meaning of the Pharmacy Act, 1868, as amended by the Poisons and Pharmacy Act, 1908, and ought to be deemed poisons in Part II. of the Schedule of Poisons to that Act. The effect of this resolution is that veronal and the other substances named and included in it can only be sold by a qualified pharmacist, and that all of these substances must be labelled "Poison," and must bear the name and address of the vendor.

The dangers from veronal poisoning are to some extent safeguarded by this valuable addition to our legislature, but the best safeguard of all will be a loyal coöperation of pharmacists with the medical profession in protecting the poblic from the abuse of drugs which have a potent though aseful therapeutic action.

Welbeck-street, $W$

\section{A CLINICAL STUDY OF THE CHIEF FUNCTION OF THE PERITONEUM. ${ }^{1}$}

BY JOHN HOWELL, M.B., B.S. LOND., F.R.C.S. ENG., HUNORAKY SURGEON TO THE CHELTENHAM GENERAI HOSPITAL, ETC.

THE chief function of the peritoneum is that of a "perienteric trap for the reception and annihilation of microorganisms which constantly and normally (i.e., without producing symptoms) permeate the intestinal wall." It is part of the duty of certain organs intimately connected with the peritoneal cavity, of which the liver, stomach, and the intestines are the chief, to excrete the toxic products formed therein, including, in times of stress, the bacteria themselves, with the minimum risk of contamination to the remainder and more important parts of the body.

In animal life the coelom is next in antiquity to the archenteron or primitive alimentary canal. When the organism consisted only of an outer layer, ectoderm, protective, sensitive, and reproductive, and an inner, endoderm, digestive and absorptive, the even distribution of nutritive material was a simple matter. When. however, a third layer, mesoderm, was developed, primarily for support and locomotion, secondarily for reproduction, any further progress in usefulness of this layer would be considerably hampered if its proper supply of nutriment continued to depend upon a correspondingly complex evagination of the alimentary canal amongst its cells. This difficulty would be solved by cleavage of the mesoderm into an inner thin layer, the splanchnopleure, and an outer layer, the somatopleure, with a cavity between (the coelom) containing fluid kept in motion by the movements of the organism. Not only did this cavity serve as a nutriment distributor, but it also became a receptacle for the products of metabolism; the cavity was therefore placed in direct communication with the exterior. In addition the reproductive cells were shed into its interior and thus found an easy exit. In the trochosphere larva of the polychrta we find a large perienteric cavity, which is not the colom (it is, in fact, the original blastocele), and for reasons which I cannot enter into here it is clear that none of the above functions are required of it, for it is practically a coelenterate, the mesoderm being relegated to the very hinder end near the anus. Why, then, this persistence of the blastocele? I suggest that here we get the first glimpse of the utility of a perienteric cavity as a trap for organisms. I may reasonably assume that along with the food a large number of cocci, bacteria, and protozoa would pass into the alimentary canal of this animalcule, some of them actively hostile. With a large number of these the cells of the endoderm would be

1 Paper founded upon the introduction to a discussion before the Medical Society of London, May 19th, 1913. able to deal, but it can be imagined that from the number or virulence of the invading organisms the resistance of this first line of defence would be not infrequently overcome. It is important that the contents of the canal should be rid of this contamination before they reached the hinder end, for here, surrounding the endoderm, are the mesodermic bands which contain the elements essential, not only for the development of the adult polychrte, but, in the form of reproductive cells, for that of countless numbers of annelids. Any bacteria, therefore, which succeed in defeating or eluding the endodermic cells only break into, they may even be enticed into, a lethal chamber containing lethal fluid, which frees itself of toxins through the primitive "pronephridia."

To proceed a step further on the road of evolution, let us consider the colom of the earthworm, with which you are no doubt familiar. It is strikingly large, though the distribution of nutritive material and conveyance of the products of metabolism are performed by an elaborate blood circulatory system, whilst the formation of reproductive cells has been relegated to one or two segments only. We have to explain why-(1) the coelomic cavity is relatively so large, a characteristic of this and allied types; (2) it communicates directly with the exterior by dorsal pores after the eighth segment; (3) dorsal pores are not found in the first eight segments ; and (4) in spite of the existence of a circulatory system the nephridia still open by bell-shaped ciliated mouths directly into the coelomic cavity.

To take the last first, it might be urged that the organism did not as yet depend upon the circulation entirely for the conveyance of metabolic products to the cells of the nephridial tubules, and that, even if it did, the funnels had not had time to degenerate; to which my reply is that tubules in direct communication with the exterior open by funnel-shaped mouths into the coeloms of the various orders right up to and including vertebrates, even the aminota, and this in spite of a continually improved and elaborated circulatory system. The obvious reason is that the colomic fluid contains material which is better conveyed directly to the purifying cells of the nephridial tubules than through the systemic circulation. In the case of the earthworm it might still be true that a large part of the products of metabolism is thrown directly into the coelom and then extracted by the nephridia, but even a cursory examination of the higher orders would show that in their case this could scarcely be. The poison which contaminates the colomic fluid is not the result of metabolism, but of bacteria which have permeated the intestinal wall; the cilia of the funnels gaide this fluid down the tubule; the water, or part of it, now purified, is reabsorbed by the cells of the tubules into the general circulation, so that the colomic fluid is cleaned of its poisons without risk to the body tissues. The earthworm has a partiality for what the gardener calls "fat stuff"-i.e., material teeming with bacteria of all kinds and grades of virulence-passing immense quantities of it through its alimentary tract in the course of its lifetime. I would have you believe that the single layer of ciliated epithelium and a few layers of chloragogen cells are not the only defence against the entrance of these hordes of bacteria into the body tissues. If they were, no earthworm would survive a day, and the result to the community would be appalling. Darwin has pointed out that these animals are an important agent in the formation of mould; 1 venture to add, in its purification also. On the contrary, they pass in their myriads, some killed, some maimed in the struggle with the first line of defence, into the coelomic cavity, where they are effectually dealt with by the amceboid cells and the bactericidal fluid of the coelom. The particulate relics of both sides are swept out through the dorsal pore, not down the nephridial tube, the mouth of which is guarded by cilia which prevent the blocking up by such particles of the delicate slender tubule. The coelomic fluid is purified in the way I mentioned above. This, I think, explains the large size of the colomic cavity in these scavengers, and the presence of the dorsal pore behind the pharyngeal segments, which, owing to the fact that there is no absorption by the thick-walled pharynx, and therefore no stagnation of foodstuff, would not require one. And herein lies the keynote of my theory. Around that portion of the alimentary tract where foodstuff containing bacteria is stagnated for the purpose of absorption, nature has developed a fluid-containing cavity designed for 
the reception and destruction of micro-organisms which thus find an opportunity to permeate its wall, with an apparatus for its own purification so arranged that its poisonous excretions shall not contaminate the systemic tissues. This is true of the whole range of animal life, including the highest vertebrate, man, in whom this perienteric trap, so far from degenerating, has become more complicated, yet not less efficient, than that of the earthworm. The machinery for its purification is less obvious, perhaps, only because it has been less studied.

The progressive adaptability to its environment evolved in the animal has necessitated, or at least has been accompanied by, a complex evolution of the colom as well. Its channels of excretion, direct and obvious in the lower animals, have become indirect and obscure in the higher series. The nephridia, the pronephric and mesonephric tubules gradually disappear and give place to other more elaborate and more complicated channels which, however, conform to the primitive and invariable rule that they excrete the colomic toxins without risk to the tissues of the somatopleure. The anatomical disposition of these will require time, patience, and ingenuity to unravel, to such an extent has Nature clouded their outlines in her desire to have many functions performed by one organ. In the amphioxus we see excretion carried out, on the one hand by the primitive method of tubules, on the other through a specialised portion of the body wall. Much as I should like to have done so, I do not intend on this occasion to trace the gradual evolution of these indirect channels through the rest of the vertebrate series except to hint that the original function of the air bladder of fishes was not concerned with hydro statics; that the lungs of amphibia and the air sacs of sauropsida subserve another and older purpose than that of respiration, and that when the diaphragm shut out the lungs from the mammalian colom the omentum was already in an advanced state of development; it only remained to detach its veins from the hepatic and connect them with the portal. To this day the human lung has not forgotten its most primitive function of excretion, as witness pneumonia, the bronchitis of typhoid and of gastro-enteritis, but it is now subsidiary to the omentum, par excellence the police and sanitary officer of the human colom. The human alimentary canal is constantly infected with millions of pathogenic and other bacteria. Let us follow the wanderings of an army of staphylococci which has been swallowed from, say, a suppurating antrum. For the present we will leave out of count the fact that one army follows another in rapid succession. Theoretically this army should be destroyed by the hydrochloric acid of the gastric juice, practically we know that not to be the case, otherwise no one would ever suffer from typhoid, cholera, \&c., and so it arrives in fairly good and close order in the small intestine. The valvulæ conniventes by delaying the flow of foodstuff along the canal aid in its absorption; but what excellent lurking places the recesses of their folds afford the regiments of this hostile army ! The single files of columnar epithelial cells lining these trenches, however well trained, are no match in defence against the determined onslaught of clumps of poison-forming bacteria. In the lymphoid tissue of the mucous membrane the situation becomes more critical. The leucocytes make short work of the "laggards" and "camp followers"; they drive the enemy's unassailable "squares" or clumps on to the wire entanglements of the muscularis mucosæand so break them up, finding in the process fresh opportunities for cutting off detachments.

The close-meshed net of the circular and longitudinal muscle fibres causes a further breaking up, thinning, and spreading out of the bacterial clumps, so also does the supporting tissue of the cœlomic epithelium. The bacteria are thus scattered in small and solitary groups over an extensive area by the time they arrive to give battle to their arch eneny the cells of the visceral peritoneum, trained by the inherited instincts of æons in this particular kind of warfare. Should they survive this, there still remain the serried ranks of leucocytes and the endothelial cells of the omentum, which, to change the metaphor, lies like a huge lazy parasite dining off a constant meal of organisms dutifully brought to it by the vermicular movements of the intestines. For an illustration of, this process note the spread of small tubercles in the subserous tissue around a tuberculous ulcer of the intestine. Dr. Hale White says :
"It is remarkable that tuberculous peritonitis is so rare in comparison to the frequency with which subperitoneal tubercles are found underneath tuberculous ulcers of the intestine." We know, too, how rare is peritonitis in comparison to the frequency with which we see quite extensive areas of the muscular coat denuded by ulceration of its first and second lines of defence, the mucous membrane. The secret lies in the defensive powers of the visceral endo. thelium and the omentum.

Of the omentum I may say here that this organ, or more correctly, part of an organ, has not even yet received its due meed of credit for the vitally important function it constantly performs on behalf of the mammalian community of cells. It is true that we have advanced since the days when we looked upon it as a mere storehouse for fat or as the abdominal hot-water bottle. We know it absorbs fluid and solid particles, encapsulates necrotic tissue, localises purulent collections, \&c.

Mr. Wilkie has shown in some interesting experiments how it will " prevent emigration of bacteria from devitalised bowel." But, as Schieffendecker remarks, "there exists no special organ for pathological processes," and if the omentum depended on occcasional accidents of the above kind to keep it in training its powers would be lost in the course of a generation or two. Its large blood and lymph supply, equal almost to those of the intestines and subject to the same reflex dilatation after meals, its large standing armies of phagocytes, its evolution since the days of its first appearance in the lower vertebrates, convince me that the "emergency" it is called upon to meet is a continuous one, but that its nature is not always equally critical, whilst its source is suggested by the anatomical relationship of omentum and small intestine.

It may be asked why the bacteria, during their passage through the bowel wall, do not enter the portal blood and lymph capillaries, and by these means the general circulation. Now the region in which they are most likely to do so is the mucous tissue, with its rich capillary network and large conspicuous lacteals. Their entrance into the lymph vessel is prevented by the fact that here, unlike any other region of the body, the lymph capillary or lacteal does not begin in an irregular open space, on first view the more expeditious arrangement for the absorption of fat globules, but has a club-shaped end closed in by cells which allow the entry of leucocytes bearing fat globules, but have the power of excluding undesirable passengers. The endothelinm of the blood capillary nas a similar property of selection and exclusion. But this region is policed by a dense force of leucocytes.

I take it that the immense numbers of phagocytes present on the hypoblastic portion of the gills of fishes are there, not for the purpose of devouring organisms escaping from the blood capillary, but rather of defending the delicate thinwalled vessel, which here forms part of the systemic circulation, from attack and invasion by noxious organisms in the gill stream. In this case virulence of the organism does not matter, for the phagocytes are constantly shedding themselves and their foe into the water current. In the lymphoid tissue of the intestine virulence of the invader does count and though the leucocytes shrink from engorging the most noxious of them they at least dispute their passage into the lacteal and blood capillary, render any prolonged stay in this region undesirable, and so tend to drive the intruders either back again into the intestine or into the far more fatal trap farther in, the cœlom.

In suppuratice diseases of the appendix or peritoneum how very rarely do we find suppuration of the glands drain ing the affected area; how rarely, indeed, do we find them Even enlarged. What a rare disease is pylephlebitis when one considers the opportunities. But in spite of all this micro-organisms do manage to gain entrance into the blood and lymph capillaries. The latter, on account of their size, are more likely to be invaded than the former. They are still liab]e to be picked out again by the endothelial cells, and any which escape meet their fate, in the latter case in the mesenteric glands, in the former in the liver, where the are arrested by the pseudopodia of the liver cells forming the wall of the portal capillary.

Now we have to consider the methods by which the peritoneal "shambles" are cleared of the bacterial carcasses and their ante-mortem- and endo-toxins without risk to the body tissues. At times of small or normal stress-i.e., when no 
"symptoms" are produced-the dead bacteria are either (1) entirely digested by the phagocytes of the omentum; (2) conveyed by them $(a)$ back into the intestinal lumen or (b) into the mesenteric glands by the lymph channels; (c) to the liver by the intestinal and omental blood. vessels, where they are arrested by the liver cells and cast out into the bile. The contaminated lymph of the cavity is absorbed by the visceral endothelium back into the Jymph spaces of the intestinal wall and omentum, whence it is either (a) extracted by Lieberkiihn's glands into the intestine, or $(b)$ absorbed by intestinal and omental veins and extracted, perhaps chemically altered, by the liver cells, and excreted in the bile which passes down the intestine and acts possibly as an antitoxin to that particular variety of bacterium.

How often has it happened that a surgeon when operating for a totally different abdominal condition has found miliary tuberculosis of the peritoneum which has given rise to no symptoms. Twice has this experience befallen me-one a case of ventral hernia and the other of inguinal hernia. Botb, curiously, were women. They refused my advice to take a prolonged rest in the open air, and three weeks after the operation went back to their housework. Neither has had a day's illness since. Had it not been for the accident of operation this incident in the patients' histories would never have been known, for the normal machinery was equal to the demand.

I would also allude here to the thousands of abdominal cases which in the pre-antiseptic and even the pre-glove days emerged successfully from a surgical operation with suppuration limited to the abdominal wall. The peritoneum had done its work silently and effectively. Iane's constipation bands are not evolution bands, but the scars of silent battles. Not content with this elaborate excretory machinery the portal blood and the blood which has received the outflow of the thoracic duct is conveyed over an immense purifying surface in the alveoli of the lungs before it is considered sufficiently pure to supply the body tissues. Our experience of the expiratory exhalations of certain of our friends proves this to be no superfluous precaution. Now let us take a case in which the stress upon the peritoneal organ was so great that the usual machinery I have just described, unless assisted, is not equal to the task imposed upon it.

Nature is still far from the end of her resources, but from the very fact that the machinery she falls back upon is not constantly in use, symptoms and physical signs are produced. These, so far from impressing us that physiology is "disordered" (Barnard), should serve to demonstrate to us her " extraordinary" methods of reaction to an extraordinary injury.

The symptoms and physical signs of acute miliary tuberculosis which has been caused by the rupture of a small diseased mesenteric gland or of a tuberculous focus in a Fallopian tube will first be considered in detail, with a view to discovering clues to reserve methods of defence relied upon by the peritoneum in times of crisis. To begin with, the invasion is not by permeation, but by a sudden, almost overwhelming flood of bacilli and their toxins. The overdose is thinned and spread out as rapidly as possible beneath the omentum by the quickened vermicular and peristaltic movements of the intestines. These, together with the action of the tubercle toxins upon the exquiritely sensitive parietal peritoneal cells, give rise to anything between vague abdominal discomfort and acute pain, according to the degree of toxicity of the intraperitoneal fluid and the vigour of the contractions. To dilute this toxicity and to aid in killing the bacilli, a large quantity of serum is secreted, and absorption by the visceral endothelium is checked as much as possible, hence there is ascites. But some of the toxin escapes into the general system and causes a rise of temperature, rapid pulse, and respiration. The rapid pulse is turned to good purpose by reflex stimuli from the peritoneum to produce splanchnic arterial hyperæmia. This arterial hyperæmia would result in the hurrying of toxins through the portal vessels into the hepatic veins were it not counteracted by a delayed flow in the portal system. I need not point out all the advantages to be obtained by this "Bier's treatment" on a large scale. It gives the intestine a congested appearance, but it aids greatly the secretion of serum, delays absorption, and gives time for the liver cells to extract those toxins and bacilli which are being conveyed by the portal blood.
All movements of the abdominal wall and the diaphragm would tend to squeeze the septic peritoneal contents into the lymph and blood capillaries, and to hurry their circulation through the portal vein and larger lymph vessels. To minimise this the abdominal wall is made rigid, and the respiratory excursions of the diaphragm lessened in their extent. Peristaltic contraction of the intestinal muscular coat would also tend to have the same effect, so the muscles are inhibited, not paralysed, hence the constipation. I agree with Mr. Peter Daniel when he says that sepsis Jeads to stasis; we differ, I think, only in our suggestion as to the steps of the process. The delayed circulation in the portal tributaries carries with it the disadvantage that the blood becomes over-venous, so the intestine, pro tem., is converted into a respiratory chamber for the $\mathrm{CO}_{2}$ and other waste products; hence, there is tympanites. The intestinal epithelium is here only reverting to its ancient functione.g., in colenterates. Were it not for this temporary relief the vitality of the bowel would be very seriously and rapidly, interfered with. In these circumstances, it can be imagined that the intestinal route for the excretions of toxins from the peritoneal cavity would not be a very efficient one, so another has to be found. The peritoneum of the stomach now takes on the duty, and the peptic glands do the work normally done by Lieberkühn's glands. The bowel being inbibited, another duct to the exterior must be made use of. The stomach is filled not only with the excretions of its own glands, but also with the increased excretions of the liver cells conveyed to it by the second and first parts of the duodenum. The vomiting centre in the medulla, either reflexly stimulated from the peritoneal cavity or centrally by the poisoned systemic blood, ensures a periodic expulsion of its noxious contents, hence the vomiting of large quantities of fluid. The colour of the vomited matter will depend upon the efforts which the liver cells have to make to meet the difficulties of the case. If the stress is small the hepatic centre in the medulla, which regulates the metabolic activity of the liver cells (the gljcogenic centre), whether reflexly or centrally stimulated, goads these cells to a corresponding effort only; the vomit therefore after the expulsion of food will contain light yellow bile and much mucus; under greater stress light green bile, with a more watery acid secretion from the peptic glands, and so on, until in virulent septic cases, such as peritonitis from a perforated gangrenous appendix, we see it gradually change to sage green (acid), to black, and so-called " fæcal " (alkaline towards the end). Also, what is equally important, the combined output of the liver and stomach increases with the urgency of the case, so that the last vomits are invariably copious. In order to provide the necessary fluid for the peritoneal "wash out" the other secretions are reduced, the tongue becomes dry, and the amount of urine decreased. Such, then, are some of the resources of which Nature avails herself in a peritoneal emergency. If the dose is not too large with the aid of this extra help the crisis passes, the leucocytes having in the meantime shut up the bacteria in tubercles. The ascitic fluid is drained away by diarrhoa. The excretory function of the stomach gives place to a purely secretory, so the appetite returns and the tongue cleans, the temperature and pulse become lower, and the balance of health generally is once more restored. An abdominal incision expedites matters considerably.

Now compare this picture which I have drawn of acute tuberculous peritonitis with a more toxic and septic form, due, say, to virulent organisms permeating through the wall of an inwardly directed appendix. So great is their virulence that in their passage they are actually causing necrosis of the tissues of the appendicular wall. The crisis being more urgent, the exertions of the peritoneum become correspondingly strenuous. The pair is usually more severe; the rigidity is more marked; the breathing is shallower but more frequent. The bowel is more congested; in bad cases the flow of the portal blood may be so delayed that the bowel is almost black near the seat of the original poison factory, fading off, the further rou get from it, to a purple and dull red. Relief from the $\mathrm{CO}_{2}$, \&c., is more necessary than ever, bence the very marked distension, which in turn should be more pronounced near the poison factory and lessen the further you proceed from it. (This is true in practice, for increasing distension of the intestine, if followed, will lead to the focus of disease, whether that is a seat of obstruction 
or inflammation or the exact opposite, the site of a perforation!) The vomiting is incessant and runs more quickly through the gamut of colours from yellow to dark green and brown. Its amount during the 24 hours may seem incredible. Treves mentions a case of one and a half gallons. I saw an even greater amount in a case of pneumococcal peritonitis associated with double pneumonia. To provide the fluid for this peritoneal "wash out" through the liver and stomach the peripheral arteries are constricted, hence the small hard and wiry pulse; the other body secretions are lessened, speech becomes thick, and the dry tongue is rolled about the mouth and between the lips from time to time. The secretion of urine is reduced to a minimum ; in severe cases of romiting it may even be suppressed, the vigorous work of the hepatic and peptic cells rendering any excretion by the renal cells unnecessary. Sume of the lymph of the tissues is commandeered for the same service, hence the pinched and drawn appearance of the face and the concentrated condition of the blood. When this supply threatens to fail, or perhaps before, the body fat is broken up into water and carbon dioxide. There is a large supply of this ready at hand in the abdomen, in the subperitoneal and retroperitoneal tissues, the omentum, and the appendices epiploicr. One pound of fat yields a little more than a pint of water when completely oxidised. In very toxic cases the wasting is very rapid, the fingers and hands look gaunt and thin, the eyes fall back in their sockets. The carbon dioxide accumulates in the blood, giving it the appearance almost of tar, increasing the frequency of the respirations, setting the alæ nasi to work, colouring the lips a livid purple and the countenance an ashen grey.

With the whole body engaged in a death-grapple with a determined enemy, every nerve fibre occupied in transmitting calls from the hard-pressed peritoneum to the other organs for some help in this desperate struggle, with the higher cerebral centres subconsciously aware that the most vital medullary centres, the cardiac, respiratory, vomiting, and hepatic, are working at their highest pressure, it is not to be wondered at that the patient has an "anxious look," is restless and sleepless.

You can abolish most of the above complicated reflex reactions with a good dose of morphia, but not-and there's the rub-the stimulus which produced them. To quote Battle and Corner: "Morphia can convert a livid vomiting and distressed patient with rapid pulse and respirations into a very comfortable patient with a slow pulse, slow respirations, moist skin, moving abdominal wall, painless and not necessarily contracted abdomen." To add another from Treves's "Intestinal Obstruction": "The amount of urine if lessened increases; the vomiting becomes less troublesome or ceases for a while; stercoraceous vomiting even may cease and on the reappearance of the symptom the ejected matter may be non-fæculent."

The raison d'etre of the elaborate network of nerve fibres governing the viscera contained in the peritoneum is not far to seek; the direct connexion of the liver, stomach, and higher reaches of the peritoneum with the medulla suggests that in importance they are on a par with the heart and lungs. Surgeons have long ago learnt that to put this beautifully designed and intricate gear out of action with a dose of morphia is not a wise proceeding in cases where the peritoneal sac is infected, though we still read of the most persevering attempts to control the vomiting with other drugs, happily without any success hitherto.

I have already said that the colour, reaction, and amount of the vomit depend upon the degree of toxicity of the fluid in the peritoneal cavity; they are, therefore, reliable data not only in the diagnosis but still more in the prognosis of pathological conditions within the abdomen.

With the progressive poisoning of the peritoneal cavity causing increased demand upon the excretory powers of the liver and stomach, the latter in turn cause a reflex dilatation of the stomach to receive their combined effusions, thus adding considerably to the discomfort from an already distended abdomen. The gradual filling of the stomach is accompanied by vague epigastric pain, nausea, belching, and great mental depression, and in some cases it was possible to foretell the imminence of a large stinking vomit by the patient's livid face becoming an even duskier due. Haguenot's and Brinton's mechanical theories of the production of fæcal vomiting are open to so many insuperable objections as to make them, in my opinion, no longer tenable.
An infected peritoneum does not always cleanse itself $v i \hat{a}$ the stomach ; sometimes the intestinal route is made use of, as in puerperal fever or pneumococcal peritonitis, or they may be used alternately, diarrhœea at one time predominating, at another the vomiting ; or, again, as in very virulent infections, both channels may be used to the utmost at the same time, as in Asiatic cholera. I am aware that the accepted view of "the excessive excretion of fluid into the intestine" in this disease is that it "is due to the paralysis of the splanchnic nerves by a toxin formed by the cholera vibrio in the intestine." My suggestion is that the toxin formation takes place in the peritoneum and that the excessive secretions are meant to get rid of it, and the fact that pathologists remark upon the condition of the peri. toneum "sticky" (Osler, Wilks, and Moxon), "has a soapy feel" (Cohnheim), lends it some support.

The most frightful instance of post-operative peritoneal sepsis that I ever saw died in four days with choleraic symptoms. I even go so far as to suggest that the symptoms of typhoid fever are due to the infection of the peritoneal cavity by the B. typhosi, the sloughing l'eyer's patches being marks of their disputed but victorious passage. Once these barriers were ravaged and passed, I utterly fail to see what could possibly hinder this motile bacillus from entering the peritoneal cavity. A boy's bilious attack is due to peritoneal infection, also the summer diarrhoea of children. Some of the cases of "vicious circle" after gastro-jejunostomy are instances of septic peritonitis. Some of the failures of gastro-jejunostomy are due to the fact that the operation has been performed, not on account of organic pyloric obstruction or gastric or duodenal ulceration, but in eases of chronic infection of the peritoneal cavity, the source of which was not found; the poison therefore continuing, the liver and stomach continued their necessary work of excretion. The abdominal incision and manipulation of the viscera, by temporarily draining the peritoneum and causing a fresh sharp effusion of serum, ensured them a short respite.

The liver and stomach by no means escape themselves scot free in the performance of these vital duties. In cases of acute infection the liver cells, especially those in the outer part of the lobule, on which the greatest stress would fall, show fatty degeneration; in the more chronic forms they die out entirely, and their place is taken by fibrous tissue. Again, the muscular coats of the stomach, constantly bathed in toxic lymph passed into it by the visceral endothelium, would gradually become atonic, causing a chronic dilatation of the organ ; sometimes this lymph would actually contain organisms which the peritoneum and omentum had imperfectly dealt with, and these again, during the time that they found themselves in the submucous tissue, would produce necrosis, and begin the mischief of ulceration. In cases of still greater stress upon the peritoneum, as in some cases of puerperal fever, ruptured gangrenous gall-bladder, \&c., the organisms set up a general condition of acute phlegmonous gastritis.

The symptoms of "acute intestinal obstruction," as given in the text-books, are not symptoms of obstruction at all. The only symptoms of " obstruction "are paroxysms of acute colicky pain, due to forcible peristalsis, combined with the few reflexes which such a non-toxic stimulus would temporarily produce, and which I need not mention here, except to say that they would be equally characteristic of the passage of a gall-stone, renal calculus, \&c. The other symptoms are due, not to the obstruction, but to the supervening poisoning of the peritoneal cavity by organisms now permeating in excessive numbers through bowel wall, the vitality of which had been depressed. Such depression would be produced not only behind a growth or other obstruction, but by interference with its blood-supply, such as embolism of a mesenteric artery, thrombosis of a mesenteric vein, and aneurysms of the abdominal aorta, from injuries to the abdomen, such as blows, run over, \&c. Excessive permeation might conceivably take place from the presence of excessive numbers within the bowel, even without any alteration in its wall, and it is not an uncommon experience to have a case die with the symptoms of acute intestinal obstruction where nothing can be found post mortem.

My labour will not have been in vain if it causes you to look upon the peritoneum with more questioning eyes. Before venturing to address so learned a society I had fortified myself with a passage from Henle: "It is an important fact that in the whole extent of this matter there is scarcely an opinion which is not founded on experience 
and again contradicted by experience. In these circum. stances one might advise theory to wait until experience is riper, but I rather think that experience in order to ripen needs the light of a reasonable theory: for Nature only answers when she is asked a question, or more truly she speaks to us constantly and with a thousand tongues, but it is only the answer to our question we hear."

Cheltenham.

\section{THE EXPERIMENTAL TRANSMISSION OF DISSEMINATED SCLEROSIS TO RABBITS.}

BY W. E. BULLOCK, M.D. EdIN., B.Sc. LoND., FORMERLY MCCUNN SCHOLAR AND ASSISTANT TO THE PROFESSOR OF PATHOLOGY, UNIVERSITY OF EDINBURGH ; ASSISTANT, IMPERIAI CANCER RESEARCH FUND, LONDON.

THE naked-eye and microscopic anatomy of the lesions found in multiple sclerosis have been studied with great care by many histologists, and especially by Dr. James Dawson; but so far as I am aware there are no recorded experimental investigations on the etiology of the disease. It is beside the purpose of this short article to analyse the cause of this, though it may be suggested that it is partly due to the more or less general belief that the disease is due to some inherent defect of the nervous system (Strümpel, Ed. Müller). Among many other factors believed to be effective in the causation of the risease one may mention syphilis (Cotala, PerrinParisot), the various acute infectious diseases (Marie), chronic poisoning with metals or carbon dioxide (Oppenheim), the puerperium (Balient), and trauma (Mendel). The experiments to be described below afford some evidence that the disease is due either to an infection by an ultramicroscopic organism or to a poison which may be found in the cerebrospinal fluid.

One of the chief difficulties which beset any experimental investigation into the cause of disseminated sclerosis is the fact that it is impossible to obtain freshly attacked nervous tissue. Owing to the chronicity of the disease one may assume that the amount of virus remaining in the central nervous system at the time of death is exceedingly small. There remains, however, the possibility of withdrawing cerebro-spinal fluid by lumbar puncture during life and inoculating this into animals, as well as the general method of testing the toxicity of the blood, urine, and the Berkefeld filtered water-soluble constituents of the frees. The first two of these methods were employed in the manner described below.

The cerebro-spinal fluid was obtained from a patient aged 19, a domestic servant, who was under the care of Dr. J. J. Graham Brown in the Royal Infirmary, Edinburgh. At the time the first specimen of the fluid was withdrawn the patient exhibited all the classical signs and symptoms of the disease. She was unable to stand owing to spastic paralysis; her speech was staccato; muscular coördination was defective and nystagmas was marked. She suffered from paralysis of the right external rectus ocular muscle and a right-sided facial paralysis. Optic atrophy was present in both eyes. She complained of cramps and pains in the arms and legs. Increased knee-jerks and Babinski's sign were present.

The fluid obtained was placed in an ice chamber within five minutes of its withdrawal and kept there for 24 hours. Part of it was then injected into a tame rabbit and a cat.

In the case of the rabbit 2 c.c. were injected subcutaneously in the line of the left sciatic nerve, whilst the cat received 2 c.c. intradurally in the thoracic region, the dura being exposed under ether anzsthesia. Tubes of broth, agar, glucose agar, blood agar, and gelatin were inoculated from the remainder of the fluid, which was then replaced in the ice chamber. The culture media showed no growth after 10 days' incubation.

After the injections the animals were isolated from laboratory stock and well tended. Apart from a slight conjunctivitis the cat remained in perfect health for nine weeks, and nothing more need be said concerning the animal.

The rabbit remained in good health for 12 days, but on the thirteenth dav developed within 12 hours complete paralysis of the hind limbs. On the fourteenth day the animal was much worse and suffered from incontinence of fæces and urine, the urine containing blood, bladder epithelium, and a variety of micro-organisms. The temperature of the animal was $99^{\circ} \mathrm{F}$. On the sixteenth day the animal was completely paralysed in all four limus, and appeared to be dying. It was therefore killed, and after withdrawing 6 c.c. of sterile heart's blood the whole of its central nervous system, the sciatic nerves, bladder, kidneys, suprarenal bodies, and lungs were removed aud preserved in 4 per cent. formalin. There were no signs of inflammation at the site of noculation.

The sterile heart's blood-proved to be sterile by culture tests-was injected into a young white Andalusian rabbit. The animal remained in good health for eight weeks.

The results of the examination of the organs and tissues of the paralysed rabbit were as follows. The lungs, kidneys, and suprarenal odies were healthy. The bladder showed a marked cystitis, due probably to an ascending infection. The sciatic nerves were healthy; the spinal cord as a whole was swollen and exhibited in the grey matter of

\section{FIG. 1.}

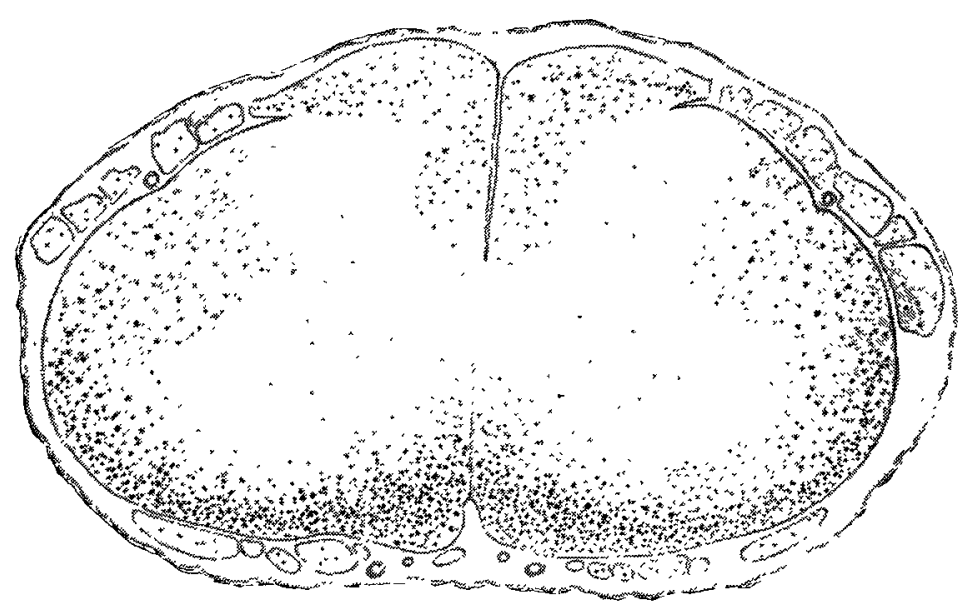

Transwerse section of spinal cord of paralysed rabbit, stained by Marchi's method, showing diffuse distribution of degenerated nerve fibres as black dots.

FIG. 2.

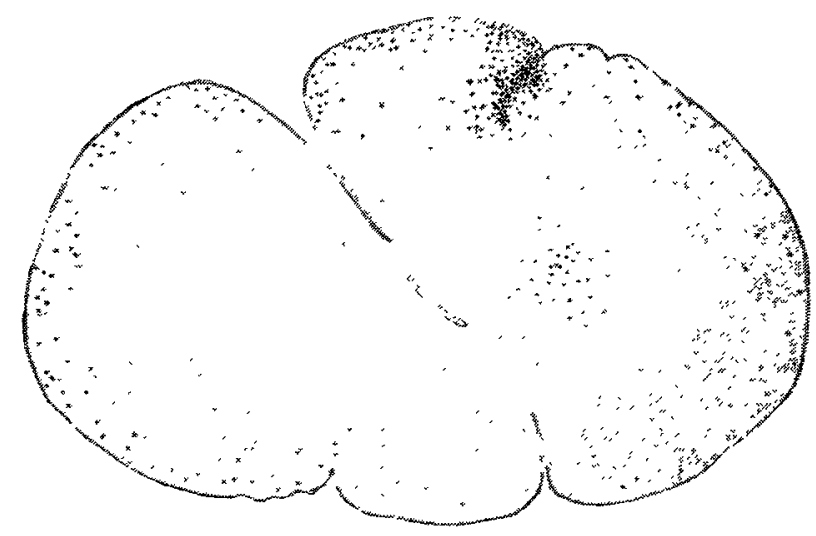

Oblique section of spinal cord of the same animal as Fig. 1 stained by Marchi's method, showing a localised area of degeneration.

FIG. 3.

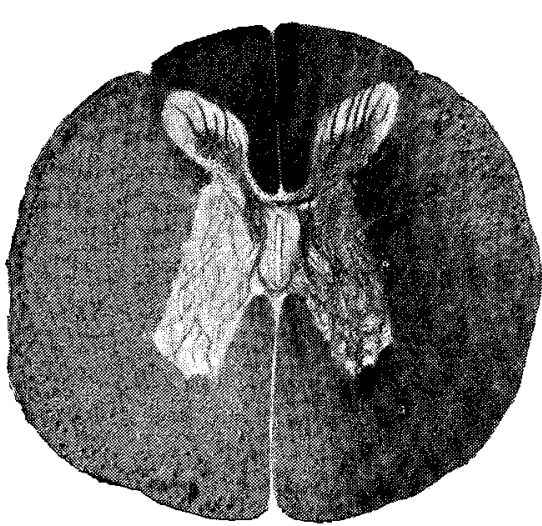

Tr.unsverse section of spinal cord of the same animal as in previous figures, stained by Weigert-Pal method, showing very extensive clegeneration (pale areas of white matter).

the lumbar and cervical regions naked eye areas of congestion. Frozen sections were made and stained with osmic acid ; fragmentation of the myelin sheath in the white matter of the cervical and lumbar regions and in several parts of the dorsal region, together with a swollen appearance of the nerve cells, was observed. Marchi preparations showed no areas of blackened fibres. In sections stained with picrofuchsin congestion of the membranes was conspicuous.

The success which attended this first inoculation of cerebro-spinal fluid led me to use up the fluid which had 